\title{
THE TRIBOLOGICAL PROPERTIES AND THE MICROSTRUCTURE INVESTIGATIONS OF TIN BABBIT WITH Pb ADDITION AFTER HEAT TREATMENT
}

\begin{abstract}
The results of tribological tests and microstructure examinations of tin babbit with $1.5 \% \mathrm{~Pb}$ addition after heat treatment are presented in this paper. The specimens were annealed at $150^{\circ} \mathrm{C}$ for $2,8,24$ and 48 hours and then tested for wear resistance and Brinell hardness. The wear tests were carried out using a block-on-ring tester operating under dry and wet conditions. The samples were also investigated by light microscopy (LM) and scanning electron microscopy (SEM).
\end{abstract}

\section{Introduction}

Bearing alloys form a special group of materials which have found wide use in the manufacture of slide bearing bushings. The bearing alloys used in commercial practice include cast irons, bronzes, and alloys based on tin, lead, zinc and aluminium. The most common alloys are those of tin and lead. They are used for lining slide bearings in cars, wagons, turbines, mills and other devices. The microstructure of these alloys consists of a soft and plastic matrix carrying the particles of hard phases that provide good abrasion resistance [1 - 6]. The best properties exhibit tin-based alloys containing in their composition $7 \div 13 \% \mathrm{Sb}, 3 \div 7 \% \mathrm{Cu}$ and up to $1.2 \%$ $\mathrm{Cd}$; they are known under the common name of tin babbitts. Their matrix is composed of fine-grained eutectic rich in tin with precipitates of hard phases like $\mathrm{Sn}_{3} \mathrm{Sb}$ in the form cubes and needle-shaped $\mathrm{Cu}_{6} \mathrm{Sn}_{5}$. Tin babbitts can carry surface loads of up to $1 \mathrm{kN} / \mathrm{cm}^{2}$ at a circumferential speed of approx. $5 \mathrm{~m} / \mathrm{s}$.

Due to the high price of tin, tin-based babbitts are used only as a lining for bearings exposed to operation under the conditions involving both high loads and high rotational speeds. To reduce the cost of tin, it is partially replaced by lead, thus allowing the tin content to be reduced to a dozen percent [2].

The tested $\mathrm{SnSb} 12 \mathrm{Cu} 6 \mathrm{~Pb}$ alloy offers good resistance to abrasion caused by rough surfaces of pivots and good sliding properties at both high and low linear velocities in the range of hydrostatic and medium loads. It is also characterized by good impact strength and shows susceptibility to bending stresses initiated by changes in the direction of rotation and edge pressure $[5,6]$. The tribological properties of this alloy can be improved, among others, by refinement and changes in the morphology of precipitates present in the alloy, as confirmed by earlier studies done by the authors of this article [5]. One of the ways to refine the precipitates present in the alloy is through a properly selected heat treatment.
The aim of these studies was to determine the influence of heat treatment on the wear properties and changes in the microstructure of the $\mathrm{SnSb} 12 \mathrm{Cu} 6 \mathrm{~Pb} 1.5$ bearing babbitt.

\section{Experimental procedure}

The investigations were carried out on the $\mathrm{SnSb} 12 \mathrm{Cu} 6 \mathrm{~Pb}$ tin babbitt alloy. The chemical composition of the high-tin $\mathrm{SnSb} 12 \mathrm{Cu} 6 \mathrm{~Pb}$ alloy is given in Table 1 .

TABLE 1

Chemical composition of babbitt alloy, wt $\%$ (according to PN-ISO 4381:1997 Standard)

\begin{tabular}{|c|c|c|c|c|c|c|}
\hline \hline $\mathrm{Pb}$ & $\mathrm{Sn}$ & $\mathrm{Sb}$ & $\mathrm{Cu}$ & $\mathrm{As}$ & $\mathrm{Cd}$ & $\mathrm{Ni}$ \\
\hline $1-3$ & balance & $11-13$ & $5-7$ & 0.1 & - & - \\
\hline
\end{tabular}

The samples were annealed under the following conditions:

- heating in furnace chamber to the temperature of $150^{\circ} \mathrm{C}$ $(423 \mathrm{~K})$ at a rate of $15^{\circ} \mathrm{C} / \mathrm{min}$,

- $\quad$ isothermal annealing at the temperature of $150^{\circ} \mathrm{C}(423 \mathrm{~K})$ for 2, 8, 24 and 48 hours,

- cooling in the furnace cooler to ambient temperature.

The heat treatment temperature was calculated from the $\mathrm{Pb}-\mathrm{Sn}$ phase diagram (Fig. 1).

In accordance with the $\mathrm{Pb}-\mathrm{Sn}$ phase diagram, at a temperature of $456 \mathrm{~K}$, a brittle eutectic is formed at the grain boundaries reducing the alloy toughness and resistance to high temperatures.

The samples were subsequently tested for wear resistance and Brinell hardness. They were also examined by light microscopy (LM) and scanning electron microscopy (SEM). The wear tests were carried out using a block-on-ring tester (Fig. 2).

\footnotetext{
* AGH UNIVERSITY OF SCIENCE AND TECHNOLOGY, FACULTY OF NON-FERROUS METALS, AL. A. MICKIEWICZA 30, 30-059 KRAKOW, POLAND

** AGH UNIVERSITY OF SCIENCE AND TECHNOLOGY, FACULTY OF METALS ENGINEERING AND COMPUTER SCIENCE, AL. A. MICKIEWICZA 30, 30-059 KRAKOW, POLAND

* Corresponding author: bleszcz@agh.edu.pl
} 


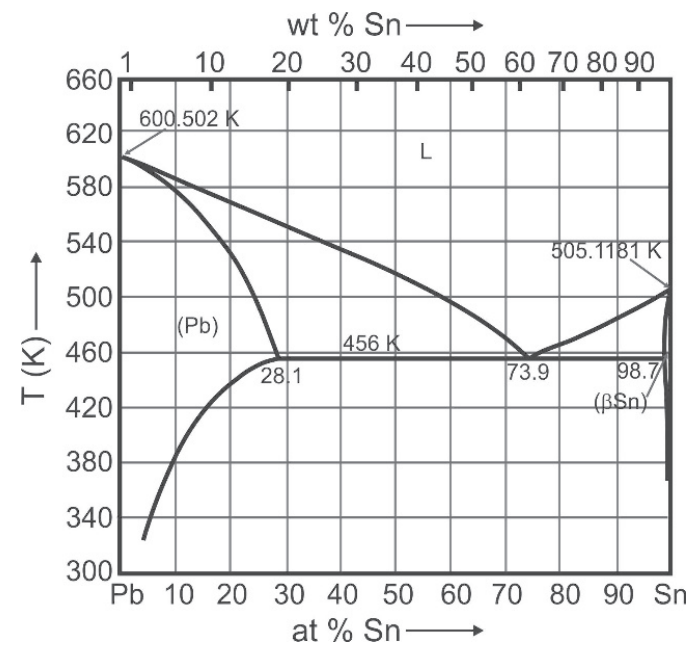

Fig. $1 . \mathrm{Pb}-\mathrm{Sn}$ phase diagram [8]

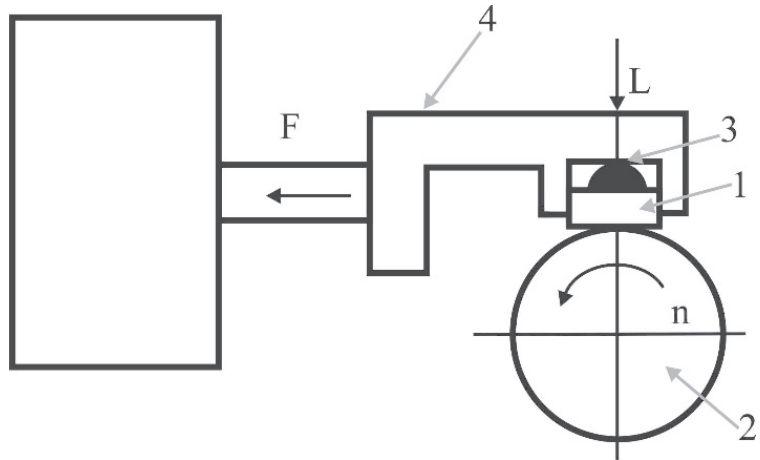

Fig. 2. Schematic view of block-on-ring tester
The sample (1) was mounted in a sample holder (4) equipped with a hemispherical insert (3) ensuring proper contact between the sample and a rotating ring (2). The wear surface was perpendicular to the pressing direction. Double wear system input the load L, pressing the sample to the ring with an accuracy of $\pm 1 \%$. The ring rotated with a constant rotating speed. The lubricant used in the wear resistance test was TU-32 oil charged from the TK-120 steam turbine during its operation.

The wear test conditions chosen for the current investigations were as follows:

- tested samples - rectangular specimens of $20 \times 4 \times 4 \mathrm{~mm}$,

- counter-specimen (rotating ring) - $\varphi 49.5 \times 8 \mathrm{~mm}$ made from the heat treated steel of $55 \mathrm{HRC}$,

- $\quad$ dry or wet sliding,

- rotational speed - $136 \mathrm{rev} / \mathrm{min}$,

- $\quad$ load $-67 \mathrm{~N}$

- $\quad$ sliding distance - dry: $100 \mathrm{~m}, 1000 \mathrm{~m}$; wet: $1000 \mathrm{~m}$, $10000 \mathrm{~m}$

The measured parameters were:

- loss of sample weight,

- friction force F (used to calculate the friction coefficient).

The tester enabled performing tests in accordance with the methods determined by ASTM D 2714, D 3704, D 2981 and G 77 Standards. The tests were performed under the conditions of dry and wet friction using a cooling-separating medium. Testing of friction with the application of a lubricating medium started under dry friction conditions.
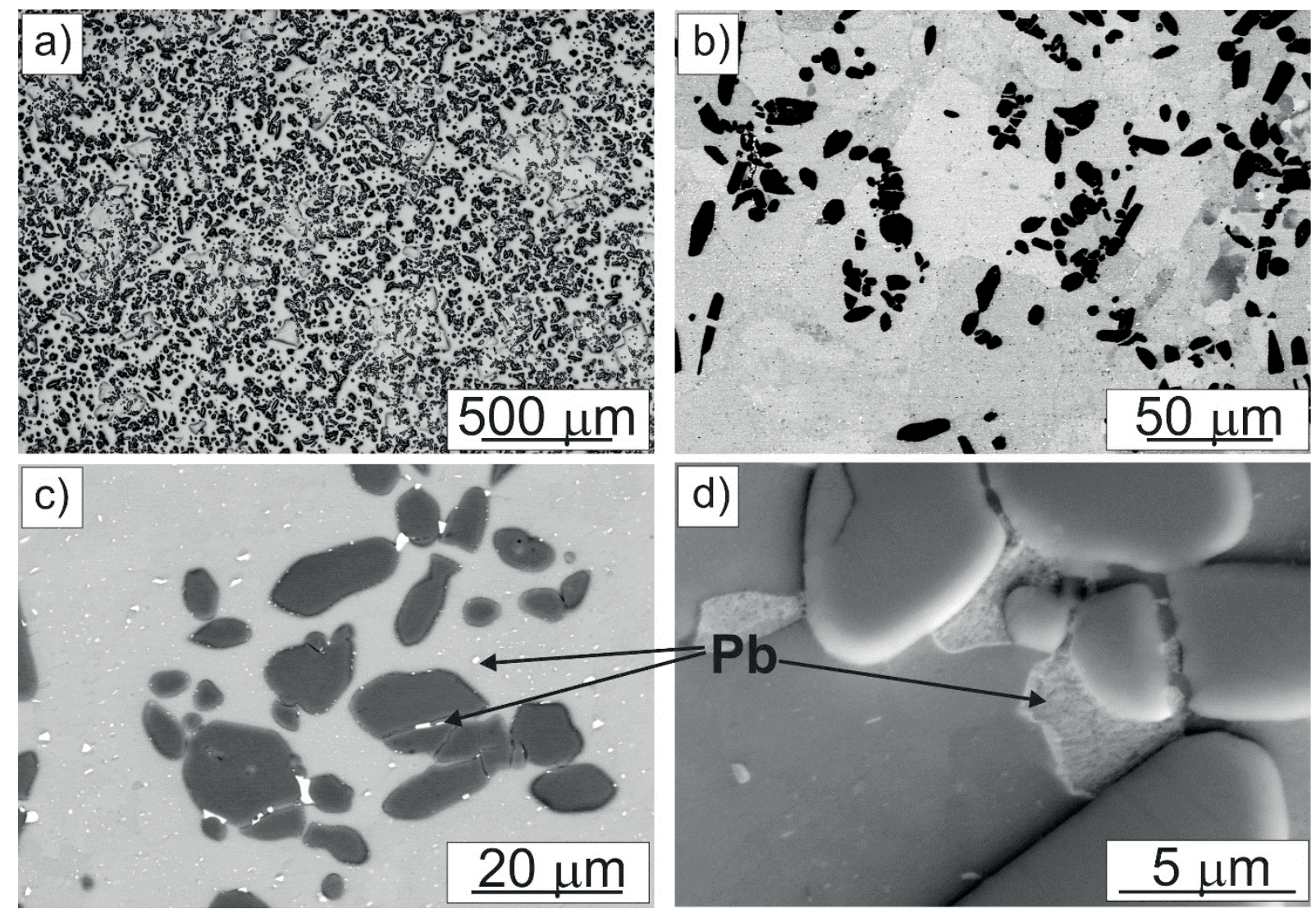

Fig.3. Microstructure of the babbitt (B82) ingot 


\section{Results}

The characteristic microstructures of the as-cast ingot are shown in Figures 3 and 4, while selected microstructures after the heat treatment are depicted in Figures 5 - 7 . Additionally, Figure 4 shows the results of point analysis and characteristic spectra. Against the background of a tin-rich matrix, phases like $\mathrm{Cu}_{6} \mathrm{Sn}_{5}$ and $\mathrm{Sn}_{3} \mathrm{Sb}_{2}$ (regular polyhedra) are visible. Locally, lead precipitates also appear (Fig. 3d), mainly in the vicinity of the $\mathrm{Cu}_{6} \mathrm{Sn}_{5}$ phase precipitates. The precipitates of the $\mathrm{Cu}_{6} \mathrm{Sn}_{5}$ phase appear in two morphological forms, i.e. in the form of spheroids and needles (Figs. 3 7). Annealing changes the morphology of these precipitates. The examinations of microstructure after annealing have indicated that with a longer time of annealing, the precipitates of $\mathrm{Cu}_{6} \mathrm{Sn}_{5}$ were occupying a larger volume in the alloy, while the precipitates of $\mathrm{Sn}_{3} \mathrm{Sb}_{2}$ were diminishing. It has further been proved that annealing refines the lead precipitates, which in the as-cast material tend to form large clusters (Fig. $3 \mathrm{~d})$. The annealing process increases the dispersion of the lead precipitates and makes their distribution more uniform. During the process of heating and holding at a temperature of $150^{\circ} \mathrm{C}$, the solubility of $\mathrm{Pb}$ in $\mathrm{Sn}$ increases quite significantly (Fig. 1). Lead diffuses into the tin matrix and precipitates during cooling in a relatively uniform manner, forming fine precipitates in a matrix rich in tin. The fine-dispersed precipitates are particularly well visible in the microstructure of alloy annealed for 24 and $48 \mathrm{~h}$.
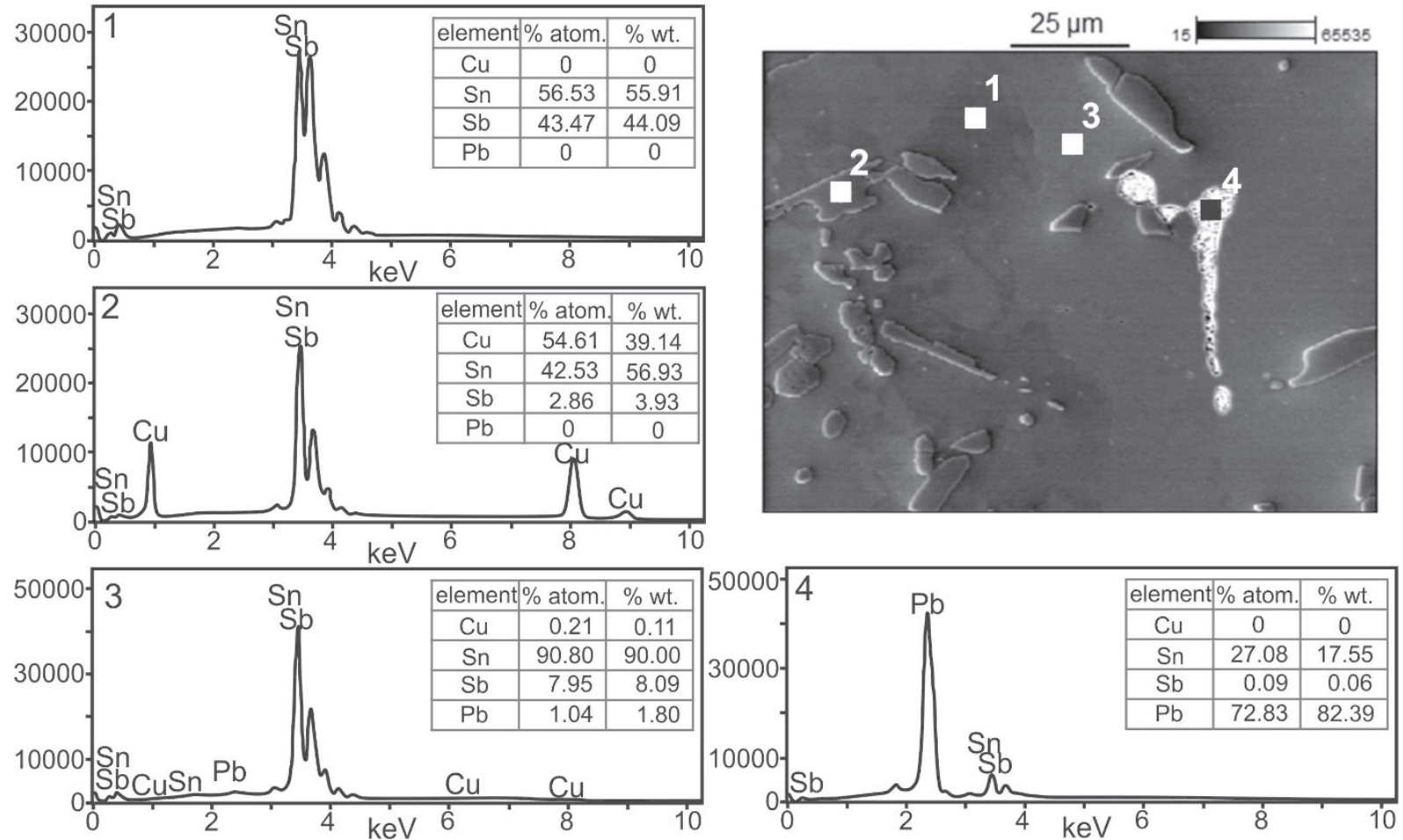

Fig. 4. Microstructure and chemical composition of phases in the babbitt (B82) ingot
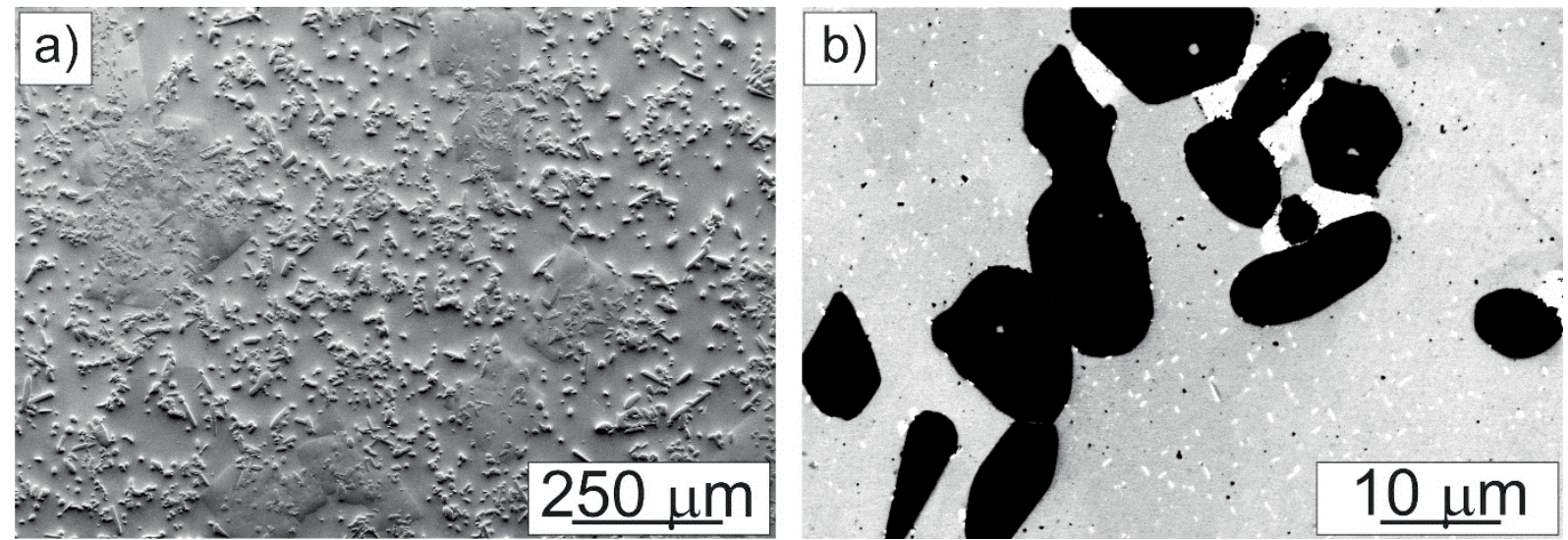

Fig. 5. Microstructure of the babbitt metal (B82) after annealing at $150^{\circ} \mathrm{C}$ for 2 hours; SEM 

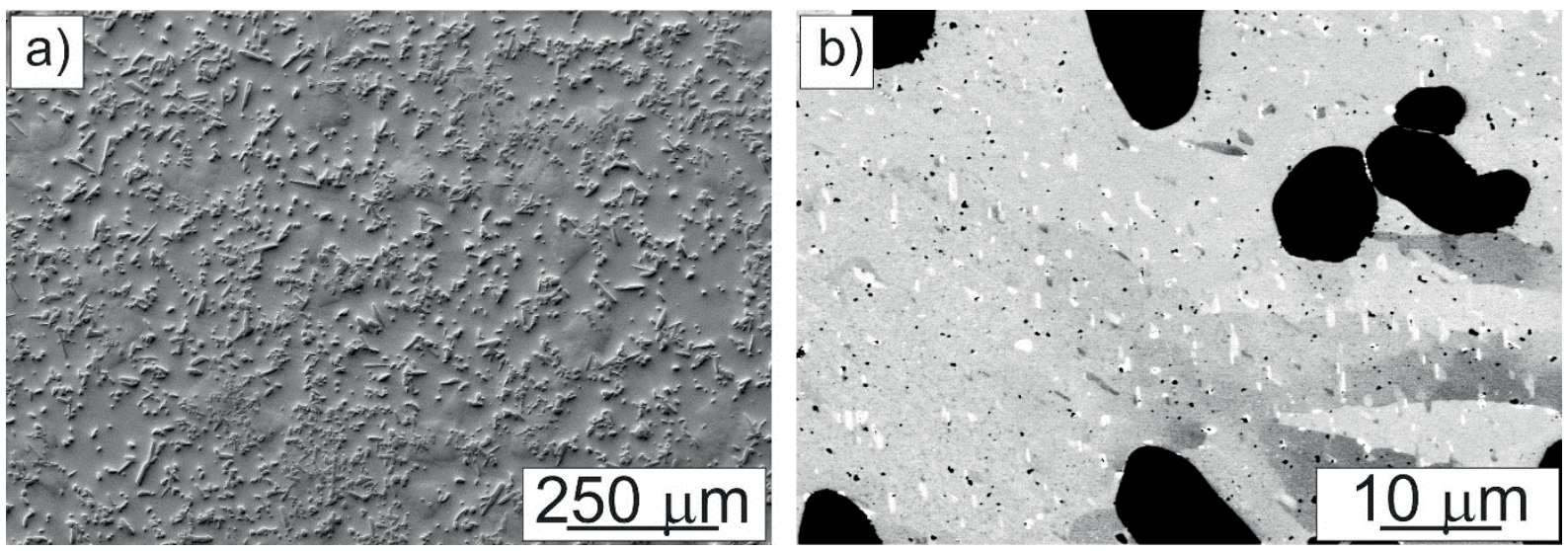

Fig. 6. Microstructure of the babbitt metal (B82) after annealing at $150^{\circ} \mathrm{C}$ for 24 hours; SEM
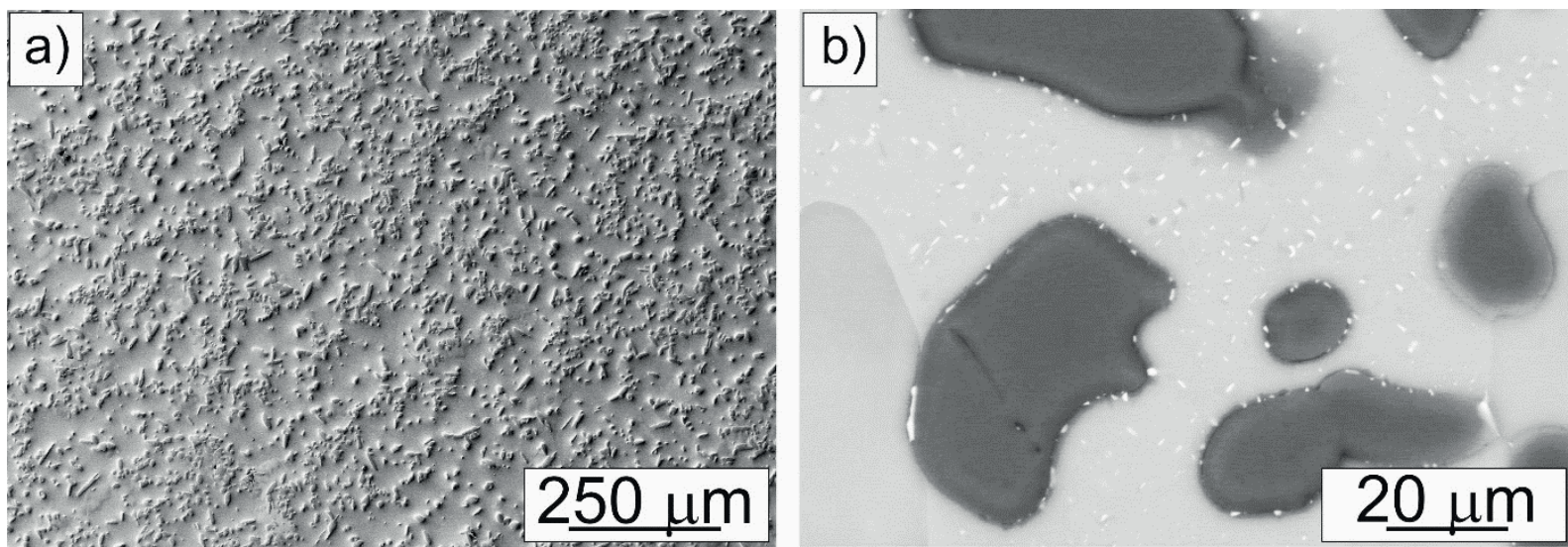

Fig.7. Microstructure of the babbitt metal (B82) after annealing at $150^{\circ} \mathrm{C}$ for 48 hours; SEM

The precipitates present in the bearing alloy occupy a large volume and during operation can cause, on the one hand, brittle fracture of the alloy, but on the other, improved abrasive wear resistance. The increasing content of fine phase precipitates in the alloy has a beneficial effect on the level of mechanical properties, as confirmed by hardness measurements (Fig. 8). The obtained results show unequivocally that longer annealing of the B82 babbitt metal leads to an increase of its hardness. The lowest hardness has the as-cast material (HB 26) and the highest has the sample after 48 hour annealing (37 HB), which means an increase by approximately $43 \%$ compared to the as-cast ingot material. The annealing for 2 hours has yielded the hardness value increased by $5 \mathrm{HB}$ compared to the as-cast ingot material, after 8 hours of annealing, the hardness was $32 \mathrm{HB}$, further prolongation of the annealing time up to 24 hours raised the hardness level to $34 \mathrm{HB}$. The increase in alloy hardness after annealing is probably due to changes in the morphology of the precipitates present in the alloy and their more uniform distribution in a matrix with high tin content.

One of the measures of the material resistance to abrasive wear adopted in the tests carried out on a T- 05 type tester was weight loss. When properly recorded, this parameter enables comparing materials in terms of their applicability for operation in the friction couples. The graphically depicted (Figs. 9 and 10) results of the weight loss, calculated as a function of the sliding distance, indicate that the weight loss increases with the increasing sliding distance. In the case of dry friction, the friction process was carried out along the sliding distance of 100 metres and 1000 metres. Under the conditions of dry friction, the largest loss of weight along the sliding distance of 100 metres was recorded in the sample after 48 hours of annealing, while for the sliding distance of 1000 metres it occurred after 2 hours of annealing (Fig. 9). The smallest weight loss along the sliding distance of 1000 metres was recorded in the sample after 48 hours of annealing, and it amounted to $0.582 \%$. Dry friction was used to monitor the phenomena that occur in the tested friction couple, for example, during the oil supply failure. Dry friction also enables determining in a much shorter time the wear mechanisms prevailing in these types of alloys. The process of friction under wet conditions was performed along the sliding distance of 1000 metres and 10000 metres. As a lubricant, TU - 32 oil, commonly used in steam turbines, was applied. Along the sliding distance of 1000 metres, the weight loss in all the tested samples was maintained at a constant level of 0.017 $0.022 \%$ (Fig. 10). Along the sliding distance of 10000 metres, the smallest loss in weight was observed in a sample after 48 hours of annealing and, related to the initial sample weight, it amounted to $0.033 \%$ (Fig. 10). The low value of the weight loss after annealing for 48 hours was certainly attributable to changes in the morphology of the precipitates present in alloy and their more even distribution in the alloy volume. The authors of [1] have found that the rate of tribological wear in alloys of this type is strongly dependent on the size of the precipitates, especially of the $\beta$ phase ( $\mathrm{SnSb})$. The heat treatment proposed by the authors of this article enables refining the precipitates present in the alloy, thus reducing the wear rate. 


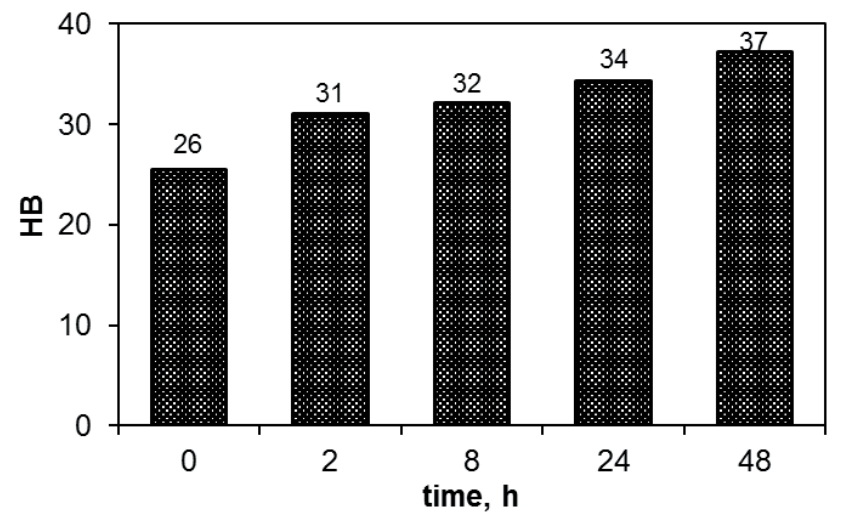

Fig. 8. Hardness of the B82 babbitt metal after heat treatment

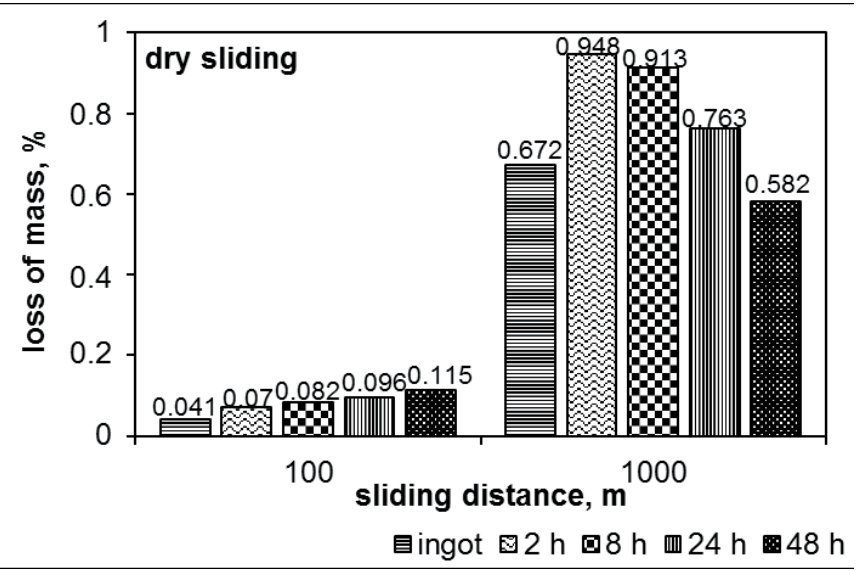

Fig. 9. Sliding distance vs weight loss in the dry friction test

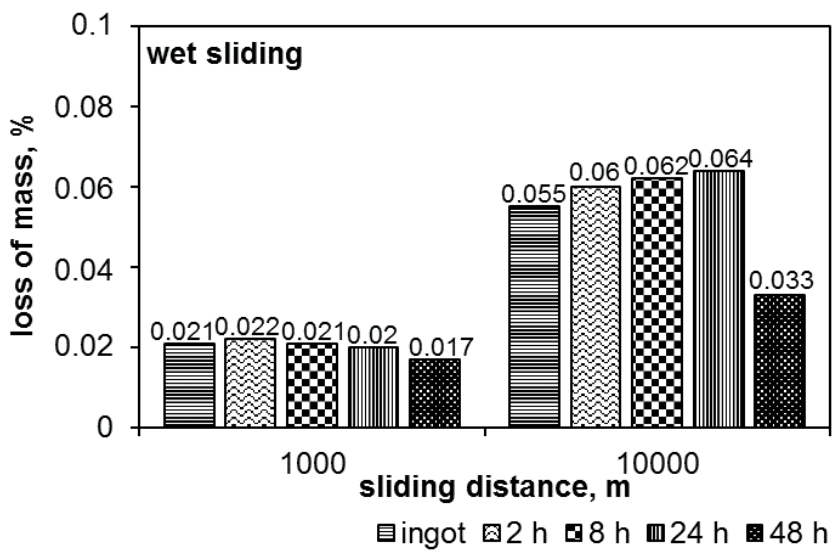

Fig.10. Sliding distance vs weight loss in the wet friction test

The coefficient of friction obtained in the process carried out under dry conditions along the sliding distance of 100 metres was in the range of $0.168-0.223$. The lowest value of 0.168 was observed in the starting sample and the maximum value of 0.223 in the sample after 8 hours of annealing. Along the sliding distance of 1000 metres, the coefficient of friction increased and was in the range of $0.274-0.588$. The change in the value of the friction coefficient was associated with changes in the geometry of friction couple and mechanism of the friction wear. The maximum value was obtained in the sample after 48 hours of annealing, while the lowest value was obtained in the material after 24 hours of annealing (Fig. 11).
Following the assumptions previously adopted, the coefficient of friction is much lower for the process carried out under wet conditions. Along the sliding distance of 1000 metres it is comprised in the range of $0.013-0.026$, the only exception being the ingot material for which the coefficient of friction has assumed the highest value of 0.102. After the annealing for 2,8 and 24 hours, the coefficient of friction was at a similar level and amounted to $0.013-0.014$. Along the sliding distance of 10000 metres, the determined coefficient of friction was in the range of $0.013-0.12$. The maximum value was obtained in the as-cast sample (Fig. 10). A slight increase in the coefficient of friction with the increasing sliding distance was associated with an increase in the tribological contact surface area and possibility of adhesive wear. The recorded coefficient of friction was low, thus indicating proper microstructure of the investigated alloy and proper selection of the sliding agent, i.e. the TU -32 oil.

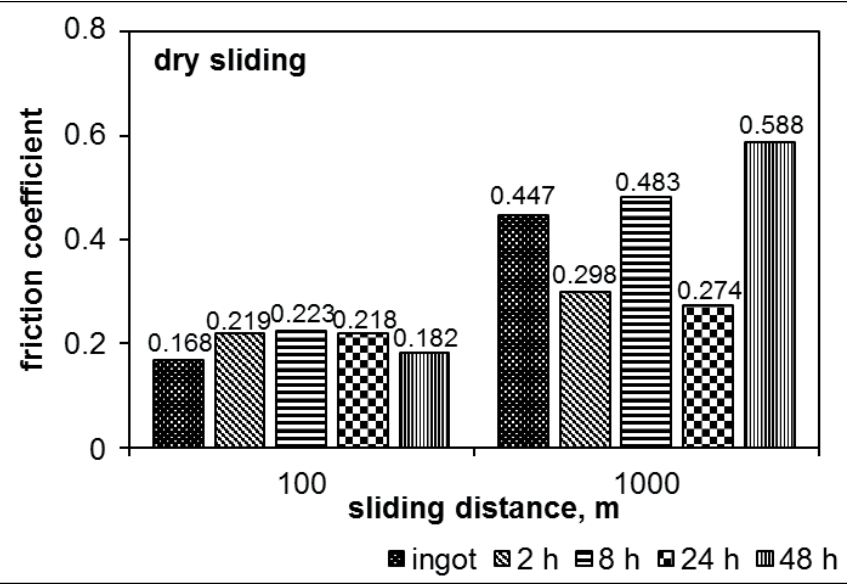

Fig. 11. Sliding distance vs friction coefficient in the dry friction test

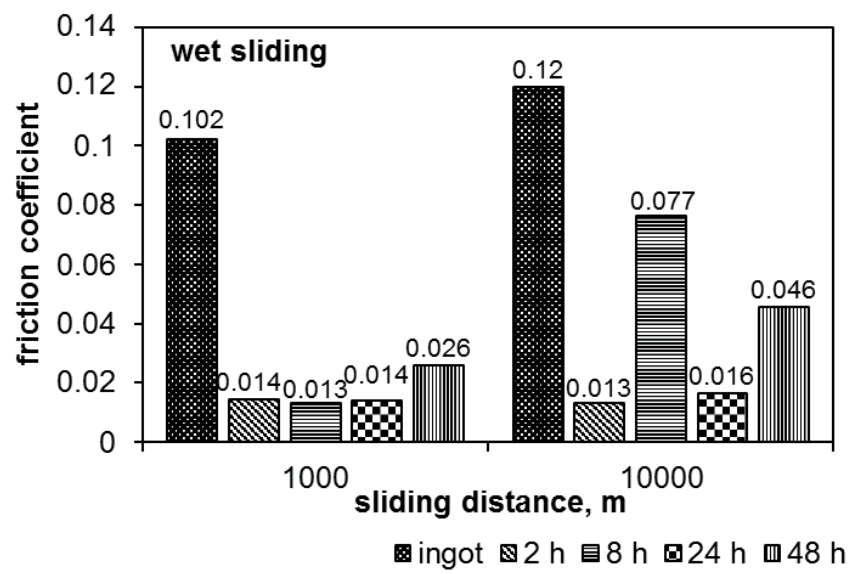

Fig. 12. Sliding distance vs friction coefficient in the wet friction test

Figures 13 and 14 show the friction surfaces of ingots in as-cast state and annealed under different conditions. For dry friction, the surfaces of samples were shown after the test carried out along the sliding distance of 1000 metres. For wet friction, the surfaces of samples were shown after the test made along the sliding distance of 10000 metres. In Figure $13 \mathrm{a}-\mathrm{d}$ showing the sample surfaces after dry friction test, one can see the removed pieces of material evidencing the adhesive type of wear; visible are also local cracks of transverse orientation 

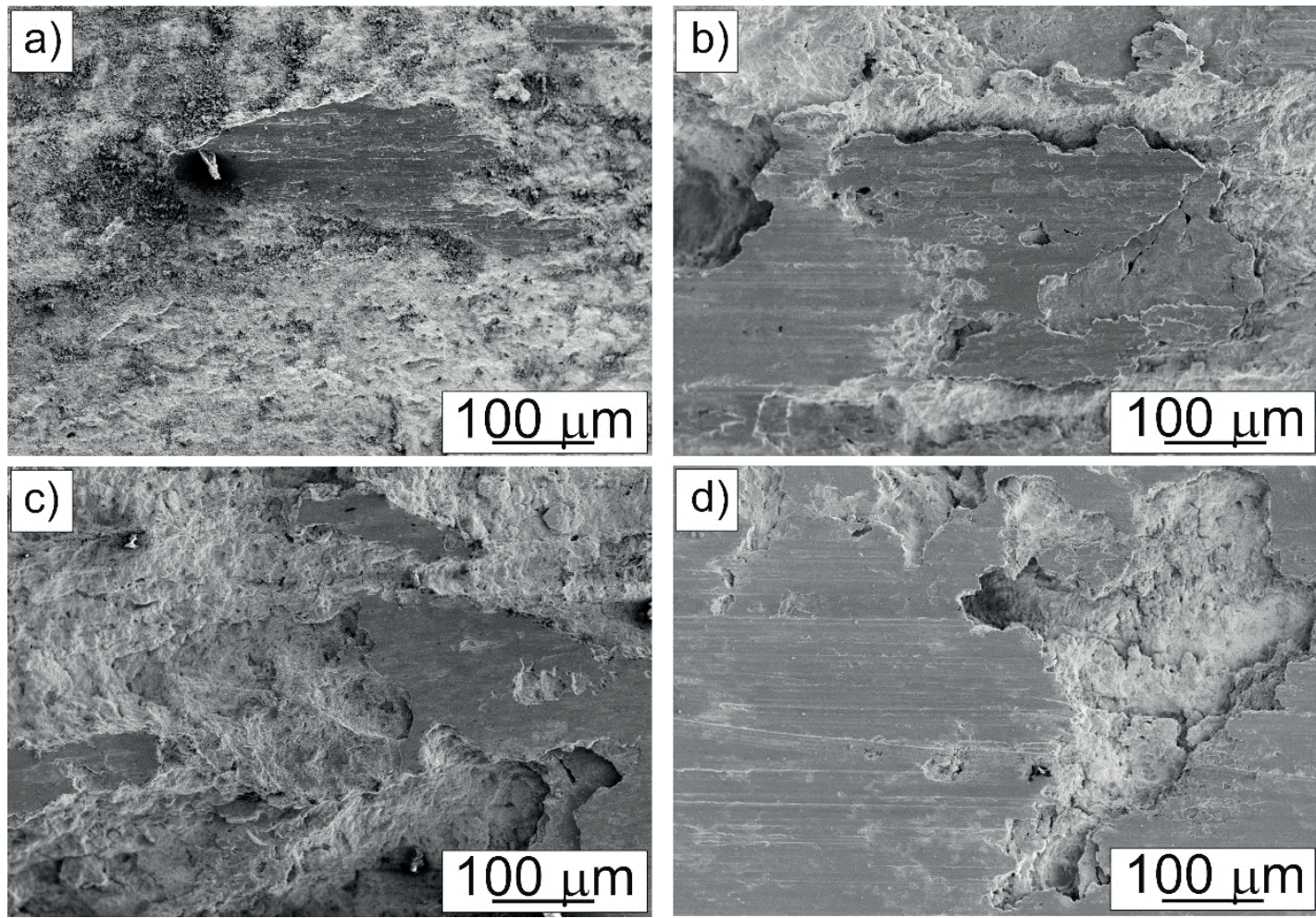

Fig. 13. Babbitt alloy surface after the dry wear resistance test carried out along the sliding distance of 1000 metres measured in ingots: a) as-cast, b) after 8 hour annealing, c) after 24 hour annealing, d) after 48 hour annealing; SEM
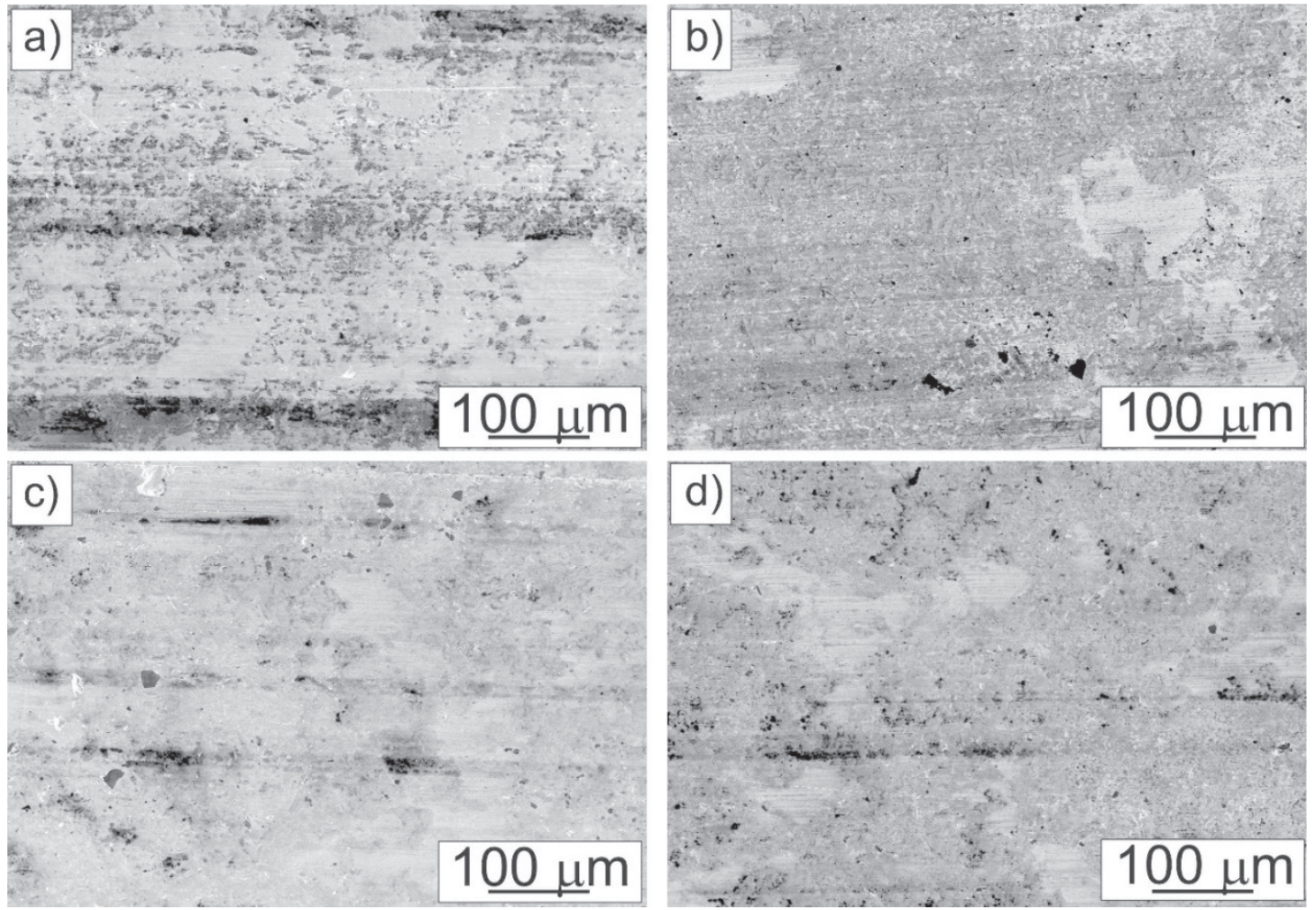

Fig. 14. Babbitt alloy surface after the wet wear resistance test carried out along the sliding distance of 10000 metres measured in ingots: a) as-cst, b) after 8 hour annealing, c) after 24 hour annealing, d) after 48 hour annealing; SEM

to the sliding distance as well as traces of abrasive wear in the form of fine cracks and fissures. The mere presence of cracks and fissures as well as their share in the overall wear of material prove the presence of abrasive mechanism and its predominant role in the initial stage of wear. However, with the sliding distance extended and changed geometry of the friction couple in the area of tribological contact, the wear mechanism changes into an adhesive type of wear. The largest share of adhesive wear was observed in the as-cast samples of ingots (Fig. 13). The surfaces subjected to friction under wet 
conditions were similar in all samples. Visible is a "smoothed surface" due to the presence of mechanisms typical of abrasive wear, which is further confirmed by the presence of numerous scratches running in the direction of the friction path. However, the share of this type of wear is very small, and its role is rather that of "smoothing" the tribological contact surface, effectively inhibited by the presence of oil lubricant. Precipitates of phases present in the alloy and local chipping effects are also observed, especially in the area of precipitate clusters present in the ingot (Fig. 14).

\section{Summary}

The conducted heat treatment promotes the refinement of precipitates present in the bearing alloy and their even distribution in a tin-rich matrix. Another effect is high degree of dispersion and more uniform distribution of lead, which in the as-cast material forms clusters detrimental to the alloy properties. The annealing temperature was selected based on the $\mathrm{Pb}-\mathrm{Sn}$ phase equilibrium diagram. To verify proper selection of the annealing temperature, the test annealing of the alloy was carried out at a temperature of $200^{\circ} \mathrm{C}(473 \mathrm{~K})$. Sample photo of the alloy microstructure is given in Figure 15. Hence it follows that the selected temperature and a long time of annealing have promoted the formation of eutectic located mainly in the vicinity of the $\mathrm{Cu}_{6} \mathrm{Sn}_{5}$ phase precipitates.

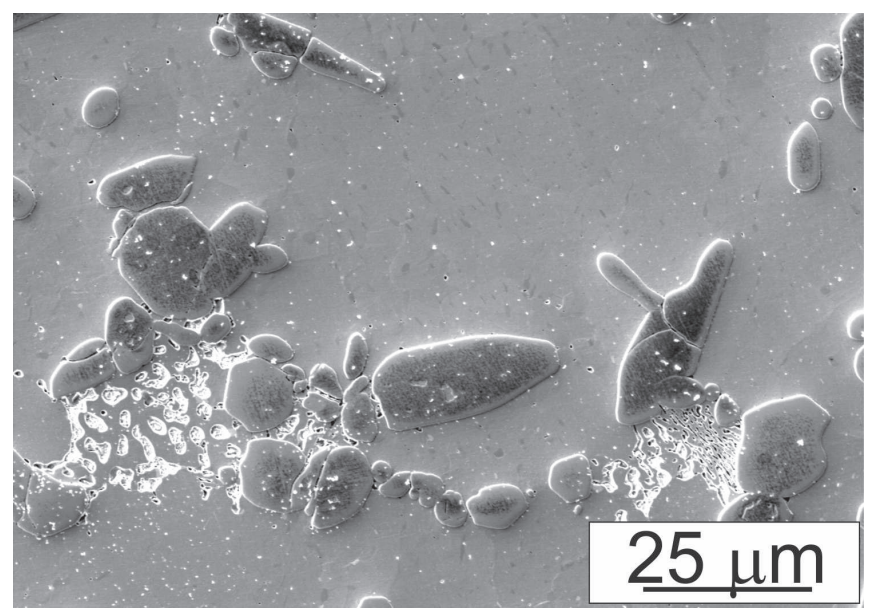

Fig. 15. Pb eutectic in babbitt metal after annealing at $200^{\circ} \mathrm{C}$ for 16 hours; SEM
The results of hardness measurements have indicated that prolongation of the sample annealing time increases the bearing alloy hardness. The highest hardness was obtained in the material annealed for 48 hours - the measured hardness value was $37 \mathrm{HB}$, which represents an increase of about $43 \%$ compared to the as-cast ingot material. The conducted studies of tribological properties show that the use of TU - 32 oil reduces quite considerably the coefficient of friction and weight loss as compared to the friction using no lubricant. The main mechanism of the surface wear during friction under wet conditions was scratching, while in the case of dry friction, the adhesive wear and fissuring were observed to prevail. From the point of view of tribological properties considered in terms of the alloy operation under wet friction conditions, the most favourable combination of properties was obtained in the alloy annealed for 48 hours. This alloy showed the lowest wear rate with the still satisfactory values of the friction coefficient.

\section{REFERENCES}

[1] N.P. Barykin, F.A. Sadykov, I. R. Aslanyan, Trenie Iznos 21, (6), $127-131$ (2000).

[2] B. A. Potekhin, V. V. Il'yushin, A. S. Khristolyubov, Materials Science and Heat Treatment 51, (7-8), 378-382 (2009).

[3] F.A. Sadykov, N.P. Barykin , I. Sh. Valeev, V. N. Danilenko, Journal of Materials Engineering and Performance 12, 29 - 36 (2003).

[4] B. Leszczyńska-Madej, M. Madej, Archives of Metallurgy and Materials 56, (3), 805 - 812 (2011).

[5] B. Leszczyńska-Madej, M. Madej, Kovove Mater. 51, 1-10 (2013).

[6] N. P. Barykin, R.F. Fazlyakhmetov, A. Kh. Valeeva, Material Science and Heat Treatment 48, (1-2), 88 - 91 (2006)

[7] PN - ISO 4381:1997 Standard

[8] B. Predel, Phase equilibria, crystallographic and thermodynamic data of binary alloys 5, Berlin Heidelberg, Springer - Verlag (1998) 
